\title{
Dietary fibre intake and risk of stroke: A systematic review and meta-analysis of prospective studies
}

\author{
D. E. Threapleton ${ }^{1}$, D. C. Greenwood ${ }^{2}$, C. E. Evans ${ }^{1}$, C. L. Cleghorn ${ }^{1}$, C. Nykjaer ${ }^{1}$, C. Woodhead ${ }^{1}$, \\ J. E. Cade ${ }^{1}$, C. Gale ${ }^{2}$ and V. J. Burley ${ }^{1}$ \\ ${ }^{1}$ School of Food Science \& Nutrition, University of Leeds, Leeds and ${ }^{2}$ Centre for Epidemiology \& Biostatistics, \\ University of Leeds, Leeds, LS2 9JT, UK
}

Stroke is a leading cause of mortality worldwide and is a major source of disability in developed countries ${ }^{(1)}$. Dietary fibre intake has been associated with stroke risk reduction in several studies but, to our knowledge, no meta-analysis of data has been previously undertaken. We have conducted a systematic review and meta-analysis to synthesize the results of existing studies. Medline and several other databases were searched for prospective studies of intake of dietary fibre and fatal or non-fatal stroke up to May 2012. Summary relative risks (RRs) were calculated using a dose response, random-effects model.

Seven cohort studies from the United States, Northern Europe, Australia and Japan were included in the meta-analysis. Total dietary fibre intake was inversely associated with stroke risk (the summary RR for a 7 g/day increment was $0.93,95 \%$ confidence intervals $(\mathrm{CI})$ 0.88 to 0.98 ) although there was some evidence of heterogeneity between studies ( $\mathrm{I}^{2}=59 \%, 95 \% \mathrm{CI}, 7 \%$ to $82 \%$ ) (Fig. 1 ). Results were similar when stratified by gender, cohort location and whether fibre was reported as non-starch polysaccharide or total dietary fibre. Using data from 4 prospective studies, the summary risk estimate in association with water-soluble dietary fibre per $4 \mathrm{~g} / \mathrm{d}$, was $0.94(95 \%$ CI 0.88 to 1.01$)$. The estimate of heterogeneity between studies was low $\left(\mathrm{I}^{2}=21 \%, 95 \% \mathrm{CI}, 0 \%\right.$ to $\left.92 \%\right)$. Heterogeneity between studies was too high to provide a meaningful pooled estimate for intakes of insoluble dietary fibre.

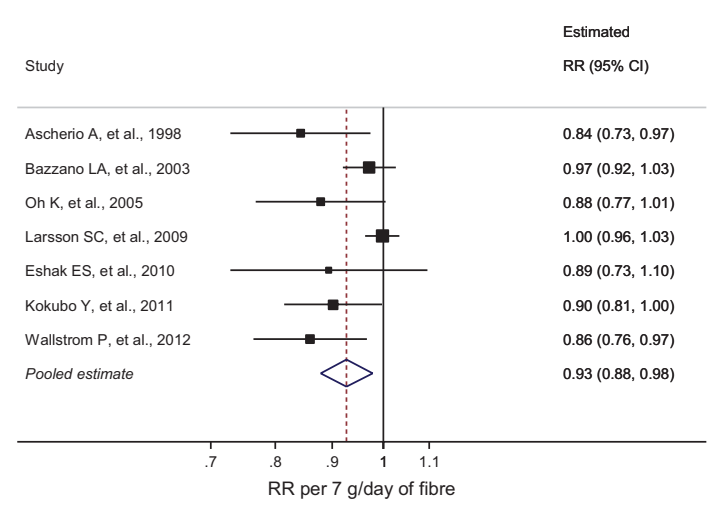

Fig. 1. Forest plot.

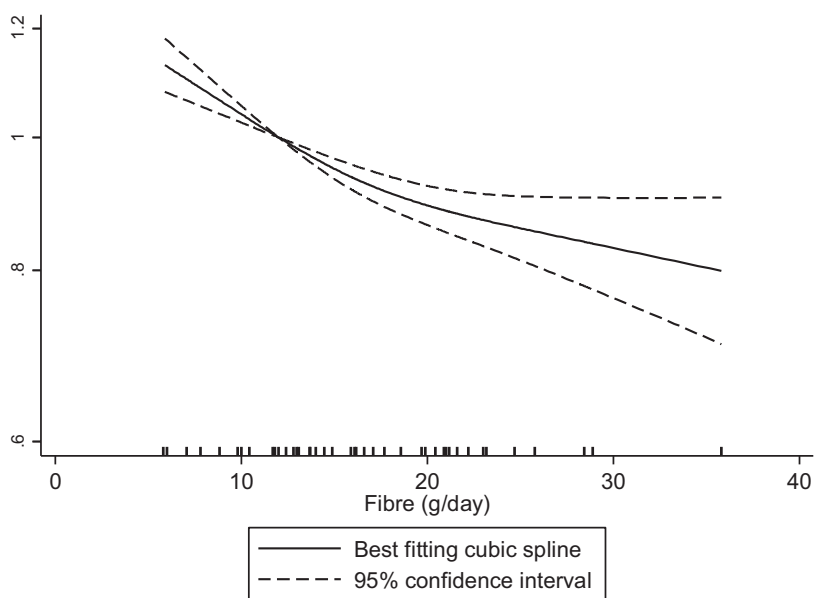

Fig. 2. Restricted cubic spline.

Each $7 \mathrm{~g} /$ day increment in dietary fibre was associated with a $7 \%$ reduction in risk of stroke, although the confidence interval around the summary estimate was wide above intakes greater than $25 \mathrm{~g} / \mathrm{d}$ (due to a smaller number of studies reporting higher intakes) (Fig. 2). Findings support recommendations to increase total dietary fibre consumption but the paucity of data on dietary fibre from different foods in relation to stroke mean conclusions cannot be drawn concerning the optimal dietary sources of dietary fibre.

This work was part funded by the Department of Health for England. DH was in receipt of a studentship from Kellogg's UK.

1. Strong K, Mathers C, Bonita R. Preventing stroke: saving lives around the world. Lancet Neurol 2007 February 6(2), $182-187$. 